\title{
Pneumothorax bei COVID-19-Erkrankung - Inzidenz und klinische Merkmale
}

\section{Massa Zantah Eduardo Dominguez-Castillo Ryan Townsend Fusun Dikengil Gerard J. Criner}

Department of Thoracic Medicine and Surgery, Lewis Katz School of Medicine at Temple University, Philadelphia, PA, USA

\section{Schlüsselwörter}

Pneumothorax $\cdot$ COVID-19. Spontanpneumothorax

\section{Zusammenfassung}

Hintergrund: Der Spontanpneumothorax ist eine selten auftretende Komplikation der Viruspneumonie bei COVID-19. Die genaue Inzidenz sowie die Risikofaktoren sind weiter unklar. In der vorliegenden Arbeit untersuchen wir die Inzidenz und die Outcomes von mehr als 3.000 Patienten mit Pneumothorax, die mit Verdacht auf COVID-19-Pneumonie in unsere Klinik aufgenommen wurden. Methoden: Wir überprüften retrospektiv die Fälle von COVID19-Patienten, die in unsere Klinik aufgenommen worden waren. Zur Berechnung der Inzidenz dieses Ereignisses wurden Patienten mit diagnostiziertem Spontanpneumothorax identifiziert und ihre klinischen Merkmale wurden umfassend dokumentiert. Es wurden Daten zum klinischen Outcome erhoben. Die einzelnen Fälle werden jeweils in Form einer kurzen Zusammenfassung vorgestellt.

Ergebnisse: Zwischen 1. März und 8. Juni 2020 wurden 3368 Patienten mit Verdacht auf eine COVID-19-Pneumonie in unsere Kli- nik aufgenommen; von diesen wiesen 902 Patienten einen positiven Nasopharyngealabstrich auf. Es wurden sechs COVID-19-Patienten, die einen Spontanpneumothorax entwickelten, identifiziert $(0,66 \%)$. Die Baseline-Bildgebung zeigte bei diesen Patienten diffuse bilaterale Milchglastrübungen und Konsolidierungen, überwiegend in den posterioren und peripheren Lungenregionen. Vier der sechs Patienten wurden mechanisch beatmet. Bei allen Patienten war eine Thoraxdrainage erforderlich. In allen Fällen bestand kein direkter Zusammenhang zwischen dem Pneumothorax und der Mortalität (66,6\%).

Schlussfolgerung: Der Spontanpneumothorax ist eine seltene Komplikation der Viruspneumonie bei COVID-19, die auch ohne mechanische Beatmung auftreten kann. Kliniker sollten im Hinblick auf die Diagnose und Behandlung dieser Komplikation wachsam sein.

(c) 2020 The Author(s)

\section{Einführung}

Der Begriff Spontanpneumothorax bezeichnet das Vorhandensein von Luft im Pleuraspalt, das nicht Folge eines Traumas oder eines anderen erkennbaren Auslösers (Verletzung oder iatrogene Ursache im Zusammenhang mit einem Eingriff) ist. Während der primäre Spontanpneumothorax ohne klinisch manifeste Lungenerkrankung auftritt, handelt es sich beim sekundären Spontanpneumothorax um eine Komplikation einer präexistenten Lungenerkrankung $[1,2]$. Bislang wird der Pneumothorax als Komplikation einer COVID-19-Viruspneumonie nur selten erwähnt, darunter einige wenige Fallberichte $[3,4,5,6]$.
Die genaue Inzidenz dieser Komplikation ist bislang nicht bekannt. In einem Bericht von Chen et al. wies 1\% (ein Patient) neben anderen radiologischen Merkmalen einen Pneumothorax auf [7]. In einer von Yang und Kollegen veröffentlichten Untersuchung von 92 verstorbenen COVID-19-Patienten hatte ein Patient $(1,1 \%)$ einen Pneumothorax, in dessen Folge er fünf Tage nach der Erstvorstellung verstarb [8]. Auch bei Patienten mit akuter MERS-CoV (Middle East respiratory syndrome corona-virus)Infektion war ein Pneumothorax mit einer schlechten Prognose assoziiert [9].

Man nimmt an, dass der Mechanismus, der dem Spontanpneumothorax bei Patienten mit COVID-19-Krankheit zugrunde 
liegt, mit den strukturellen Veränderungen im Lungenparenchym zusammenhängt. Diese umfassen zystische und fibrotische Veränderungen, die zu einer Ruptur der Alveolen führen, und treten zusätzlich zum Anstieg des intrathorakalen Drucks durch anhaltenden Husten und/oder längere mechanische Beatmung auf $[3,5,6,10,11]$.

In der vorliegenden Arbeit untersuchen wir die Inzidenz und die Outcomes von mehr als 3000 Patienten mit Pneumothorax, die mit Verdacht auf COVID-19-Pneumonie in unsere Klinik aufgenommen wurden. Wir erörtern sechs Fälle von Patienten mit COVID-19-Pneumonie, die einen Spontanpneumothorax entwickelten, und beschreiben ihre klinischen und radiologischen Charakteristika sowie die Outcomes vor dem Hintergrund anderer bisher berichteter Fälle.

\section{Methoden}

Wir überprüften retrospektiv die Krankenakten der COVID19-Patienten, die zwischen dem 1. März und dem 8. Juni 2020 in unsere Spezialklinik aufgenommen worden waren. In diesem Zeitraum behandelten wir 902 Patienten mit COVID-19. Die Diagnose wurde auf Grundlage von PCR (Polymerase-Kettenreaktion)-Testungen von Nasopharyngeal-Abstrichen gestellt. Bei allen Patienten erfolgte bei Aufnahme in die Klinik eine Computertomographie (CT) des Thorax, und täglich wurden RoutineRöntgenuntersuchungen des Thorax durchgeführt. Die Feststellung, ob ein Pneumothorax vorlag oder nicht, wurde anhand der klinischen Dokumentation und durch eine Beurteilung der Thorax-Röntgenaufnahmen getroffen. Patienten, die zu irgendeinem Zeitpunkt während des Krankheitsverlaufs einen Pneumothorax hatten, wurden einer genauen Überprüfung unterzogen. Bei jedem Patienten wurden die Baseline-Laborwerte dokumentiert, darunter die Entzündungsmarker C-reaktives Protein (CRP), Laktatedehydrogenase (LDH), Ferritin, D-Dimere, Interleukin-6, Leukozyten, absolute Lymphozyten- und Neutrophilenzahl.

Für die Beurteilung der Atemfunktion wurde der SF-Quotient herangezogen. Dieser ist definiert als Verhältnis der pulsoximetrisch gemessenen Sauerstoffsättigung $\left(\mathrm{SpO}_{2}\right)$ zur inspiratorischen Sauerstofffraktion $\left(\mathrm{FiO}_{2}\right)$ im Verhältnis zur Atemfrequenz $\left(\mathrm{SpO}_{2} / \mathrm{FiO}_{2}\right.$-Quotient). Anschließend wurde die Inzidenz des Spontanpneumothorax bei COVID-19-Patienten berechnet. Das Institutional Review Board des Temple University Hospital erteilte die Genehmigung für das Protokoll.

\section{Ergebnisse}

Im Studienzeitraum wurden mehr als 3000 Patienten mit Verdacht auf eine COVID-19-Pneumonie aufgenommen und behandelt; von diesen wiesen 902 einen COVID-19-positiven Nasopharyngealabstrich auf. Sechs Patienten entwickelten einen Pneumothorax und die Inzidenz des Spontanpneumothorax betrug 0,66\%. Die Patientenmerkmale sind in Tabelle 1 und 2 zusammengefasst.

\section{Bericht über die Fälle}

\section{Fall 1}

Ein 49-jähriger Mann mit morbider Adipositas (BMI: 47,2), koronarer Herzkrankheit und Herzinsuffizienz stellte sich wegen seit einer Woche bestehendem Fieber, nicht produktivem Husten, Störung der Geruchswahrnehmung und Kurzatmigkeit vor. Der Patient hatte nie geraucht und hatte keine pulmonale Grunderkrankung. Er benötigte Sauerstoff über eine Nasensonde (SF-Quotient: 215). In der Röntgenaufnahme des Thorax (RT) fanden sich bilaterale Infiltrate in den peripheren Mittelfeldern sowie basal. Eine CT-Aufnahme des Brustkorbs zeigte fleckförmige Milchglastrübungen und Konsolidierungen mit peripherer Verteilung in beiden Lungenflügeln. Sein Nasopharyngealabstrich war positiv für SARS-CoV-2. Bei den Laborbefunden fielen eine Lymphopenie und erhöhte Entzündungsmarker auf. Er wurde ins Krankenhaus aufgenommen und erhielt Antibiotika, Kortikosteroide, Rekonvaleszenten-Plasma und Tocilizumab als Off-Label-Anwendung. Eine Woche später entwickelte der Patient eine sich zunehmend verschlechternde Hypoxie (PF-Quotient: 65) und erhielt eine High-Flow-Nasenkanüle (high-flow nasal cannula, HFNC). Zum Ausschluss einer möglichen Lungenembolie wurde ein CT-Angiogramm erstellt, das einen ausgedehnten linksseitigen Pneumothorax zeigte. Daraufhin wurde bei dem Patienten über einen offenen chirurgischen Zugang eine Thoraxdrainage gelegt und innerhalb von zwölf Stunden bildete sich der Pneumothorax zurück. Der Krankheitsverlauf wurde durch eine Verschlechterung der Hypoxie infolge einer COVID-19-Pneumonie verkompliziert, die die Einleitung einer invasiven mechanischen Beatmung und eine veno-venösen extrakorporale Membranoxygenierung (extracoporeal membrane oxygenation, ECMO) erforderlich machte. Der Patient entwickelte ein Multiorganversagen und verstarb trotz Wiederbelebungsmaßnahmen.

\section{Fall 2}

Ein 59-jähriger Mann mit Adipositas (BMI: 32,77) stellte sich wegen seit zehn Tagen bestehender Erschöpfung, Fieber, Husten und Kurzatmigkeit vor. Bei Aufnahme hatte der Patient Fieber und eine Hypoxie und er benötigte Sauerstoff mittels HFNC (SF-Quotient: 156). Bei den Laboruntersuchungen fielen eine Lymphopenie und erhöhte Entzündungsmarker auf. In der RT fanden sich multifokale bilaterale Trübungen in den Lufträumen. Eine CTUntersuchung des Thorax zeigte Areale mit Crazy-Paving-Muster, diffuse ausgedehnte gemischte Konsolidierungen und Milchglastrübungen. Sein Nasopharyngealabstrich war positiv für SARS-CoV-2. Es wurde eine Behandlung mit Antibiotika und Kortikosteroiden eingeleitet. Im weiteren Verlauf erhielt der Patient zudem Rekonvaleszenten-Plasma und Tocilizumab als OffLabel-Anwendung. Aufgrund einer persistierenden Hypoxie und erhöhter Atemarbeit unter HFNC-Therapie (PF-Quotient: 61) wurde er zwei Tage später intubiert. Zehn Tage nach der Intubation entschied man sich für eine perkutane Tracheostomie, da eine längere Intubationsdauer absehbar war. Vor dem Eingriff waren die Beatmungseinstellungen wie folgt: Atemfrequenz (AF):
40

Kompass Pneumol 2021;9:39-46 DOI: $10.1159 / 000513501$ 
Tab. 1. Demographische und klinische Merkmale von COVID-19-Patienten mit Pneumothorax

\begin{tabular}{|c|c|c|c|c|c|c|c|}
\hline $\begin{array}{l}\text { Patient/ } \\
\text { Geschlecht/ } \\
\text { Alter in Jahren }\end{array}$ & CT-Charakteristika bei Baseline & $\begin{array}{l}\text { Risikofaktoren } \\
\text { für PTX }\end{array}$ & $\begin{array}{l}\text { Zeit bis } \\
\text { zum } \\
\text { Auftreten, } \\
\text { Tage }\end{array}$ & $\begin{array}{l}\text { Ausmaß } \\
\text { des PTX }\end{array}$ & $\begin{array}{l}\text { Thorax- } \\
\text { drainage }\end{array}$ & $\begin{array}{l}\text { Zeit bis zur } \\
\text { vollständigen } \\
\text { Rückbildung, } \\
\text { Tage }\end{array}$ & Outcomes \\
\hline $1 / \mathrm{m} / 49$ & $\begin{array}{l}\text { Fleckförmige Milchglastrübungen und bilaterale } \\
\text { Konsolidierungen mit peripherer Verteilung }\end{array}$ & Keine & 11 & Groß & Ja & 0,5 & Verstorben \\
\hline $2 / \mathrm{m} / 59$ & $\begin{array}{l}\text { Fleckförmige Milchglastrübungen, Konsolidierungen } \\
\text { und «Crazy-Paving»-Muster mit bilateraler peripherer } \\
\text { Verteilung }\end{array}$ & $\begin{array}{l}\text { Mechanische } \\
\text { Beatmung }\end{array}$ & 12 & & Ja & $7-13$ & Überlebt \\
\hline $3 / w / 81$ & $\begin{array}{l}\text { Ausgedehnte Milchglastrübungen und bilaterale } \\
\text { konsolidierende Veränderungen mit besonders } \\
\text { ausgeprägter Konsolidierung im rechten } \\
\text { Oberlappen }\end{array}$ & Keine & 9 & Mäßig & Ja & $3-14$ & Verstorben \\
\hline 4/w/45 & $\begin{array}{l}\text { Ausgedehnte bilaterale Konsolidierungen und } \\
\text { Milchglastrübungen }\end{array}$ & $\begin{array}{l}\text { Mechanische } \\
\text { Beatmung }\end{array}$ & 1 & Klein & Ja & 1 & Überlebt \\
\hline $5 / w / 47$ & Bilaterale Konsolidierungen und Milchglastrübungen & $\begin{array}{l}\text { Mechanische } \\
\text { Beatmung }\end{array}$ & 12 & Klein & $\mathrm{Ja}$ & 1 & Verstorben \\
\hline $6 / w / 76$ & Bilaterale basale Konsolidierungen & $\begin{array}{l}\text { Mechanische } \\
\text { Beatmung } \\
\text { ILE }\end{array}$ & 21 & Groß & $\mathrm{Ja}$ & 1 & Verstorben \\
\hline
\end{tabular}

Tab. 2. Baseline-Laborwerte und -Lungenfunktionsmarker von COVID-19-Patienten mit Pneumothorax

\begin{tabular}{|c|c|c|c|c|c|c|}
\hline Fall & 1 & 2 & 3 & 4 & 5 & 6 \\
\hline \multicolumn{7}{|l|}{ Laborwerte/Entzündungsmarker bei Baseline } \\
\hline Leukozyten (Tsd./mm³) & 8,1 & 8,3 & 4,3 & 4,5 & 11,5 & 7,4 \\
\hline Absolute Lymphozytenzahl (Tsd./mm³) & 0,6 & 0,8 & 0,5 & 0,6 & 0,5 & 0,7 \\
\hline Absolute Neutrophilenzahl (Tsd./mm³) & 4,5 & 7,0 & 3,1 & 3,4 & 7,8 & 6,5 \\
\hline $\mathrm{CRP}(\mathrm{mg} / \mathrm{L})$ & 13,1 & 13,6 & 11,3 & 16,2 & 8,7 & 16,5 \\
\hline LDH (U/L) & 331 & 472 & 148 & 200 & 481 & 386 \\
\hline D-Dimere (ng/mL) & 695 & 788 & 662 & 993 & $1^{\prime} 164$ & $6^{\prime} 947$ \\
\hline Ferritin (ng/mL) & 676 & 775 & 419 & 268 & 834 & 446 \\
\hline IL-6 (pg/mL) & 80,37 & 70,39 & $<1,4$ & 6,69 & 11,95 & - \\
\hline \multicolumn{7}{|l|}{ Baseline-Lungenfunktionswerte } \\
\hline SF-Quotient & 215 & 156 & 218 & 133 & 92 & 155 \\
\hline
\end{tabular}

16/min, Atemzugvolumen (Tidalvolumen, TV): $450 \mathrm{ml}, \mathrm{FiO}_{2}$ : $60 \%$ und positiver endexspiratorischer Druck (positive end-expiratory pressure, $\mathrm{PEEP}$ ): $10 \mathrm{~cm} \mathrm{H}_{2} \mathrm{O}$. Der inspiratorische Spitzendruck (PIP) betrug $26 \mathrm{~cm} \mathrm{H}_{2} \mathrm{O}$ und der Plateaudruck $22 \mathrm{~cm} \mathrm{H}_{2} \mathrm{O}$. Während des Eingriffs und kurz nach der Platzierung des Endotrachealtubus kam es zu einem allmählichen Abfall der Sauerstoffsättigung und der Patient wurde hämodynamisch instabil. Am Beatmungsgerät wurden hohe Spitzendrücke beobachtet. Die Bedside-Sonographie zeigte eine bilateral fehlende Verschieblichkeit der Lunge. Wegen des Verdachts auf einen Spannungspneumothorax wurden bei dem Patienten über einen offenen chirurgischen Zugang bilaterale Thoraxdrainagen gelegt. Daraufhin kam es zu einer Besserung der Hämodynamik und der Oxygenierung. Anschließend wurde erfolgreich ein Endotrachealtubus (ETT) gelegt. Die postprozeduralen Röntgenaufnahmen des Thorax zeigten ein subkutanes Emphysem, jedoch keinen Restpneumothorax. Nach einer Woche wurde der Patient von der Beat- mung entwöhnt. Schließlich wurden die Thoraxdrainagen entfernt, und er wurde in eine Akut-Rehabilitationseinrichtung entlassen.

\section{Fall 3}

Eine 81-jährige Frau mit Hypertonie und Apoplexie in der Vorgeschichte stellte sich wegen zunehmender Erschöpfung, Appetitlosigkeit und Durchfall vor. Bei den Laborwerten fielen eine Lymphopenie und leicht erhöhte Entzündungsmarker auf. Auf Grund der PCR-Testergebnisse eines Nasopharyngeal-Abstrichs, der fünf Tage vor ihrer Vorstellung erfolgt war, wurde bei ihr eine COVID-19-Krankheit diagnostiziert. Eine CT-Aufnahme des Brustkorbs zeigte bilateral diffuse fleckförmige Milchglastrübungen und Konsolidierungen in den peripheren posterioren Lungenregionen. Die Patientin benötigte initial 41 Sauerstoff über eine Nasensonde (SF-Quotient: 218). Es wurde eine Kortikosteroid- und Antibiotikatherapie begonnen und sie erhielt Rem- 
desivir (im Rahmen einer klinischen Studie). Bald nach ihrer Aufnahme entwickelte die Patientin eine zunehmende Hypoxämie und benötigte Sauerstoff über eine HFNC. In den bildgebenden Untersuchungen des Thorax fanden sich vermehrte bilaterale Konsolidierungen. Vier Tage später wurde bei ihr ein kleiner rechtsbasaler Pneumothorax festgestellt, der sich innerhalb von zwei Tagen vergrößerte. Man entschied, bei der Patientin eine Thoraxdrainage unter CT-Steuerung zu legen. Drei Tage nach Rückbildung des Pneumothorax wurde die Thoraxdrainage entfernt. Einige Tage später stieg der Sauerstoffbedarf der Patientin erneut an, und eine erneute Röntgenaufnahme des Thorax zeigte einen neu aufgetretenen ausgedehnten Pneumothorax auf der linken Seite und ein kleines Pneumothoraxrezidiv auf der rechten Seite. Es wurde eine linksseitige Thoraxdrainage gelegt, woraufhin sich der Pneumothorax zurückbildete. Bedauerlicherweise verschlechterte sich der klinische Zustand der Patientin an den folgenden Tagen weiter, und sie verstarb, nachdem Maßnahmen zur Linderung ergriffen worden waren.

\section{Fall 4}

Eine 45-jährige Frau mit unauffälliger medizinischer Vorgeschichte stellte sich wegen Kurzatmigkeit und Husten vor. Bei der Aufnahme hatte sie Fieber sowie eine Hypoxie mit einer $\mathrm{SpO}_{2}$ von $80 \%$ und sie benötigte 151 Sauerstoff über eine Nasensonde (SFQuotient: 133). Bei der Erstuntersuchung des Blutes wurden eine Lymphopenie und erhöhte Entzündungsmarker im Blut festgestellt. Der PCR-Test aufCOVID-19 fiel positiv aus. Es erfolgte eine CT-Untersuchung des Thorax, die ausgedehnte bilaterale Konsolidierungen und Milchglastrübungen zeigte. Ihr Sauerstoffbedarf stieg rasch auf bis zu $50 \mathrm{l} / \mathrm{min}$ und $100 \% \mathrm{FiO}_{2}$ über HFNC an. Sie wurde intubiert und die initialen Beatmungseinstellungen waren wie folgt: AF: 18/min, TV: $450 \mathrm{ml}, \mathrm{FiO}_{2}: 100 \%$ und PEEP: $10 \mathrm{~cm}$ $\mathrm{H}_{2} \mathrm{O}$, und der daraus resultierende PF-Quotient betrug 148. Die nach der Intubation erstellte Röntgenaufnahme des Thorax zeigte eine Intubation im rechten Hauptbronchus und eine Atelektase des linken Lungenflügels. Daraufhin wurde der ETT bis zur korrekten Position zurückgezogen und die erneute bildgebende Untersuchung ergab einen kleinen apikalen Pneumothorax auf der linken Seite. Bei der Patientin wurde über einen Thorakotomiezugang eine Thoraxdrainage gelegt, woraufhin sich der Pneumothorax zurückbildete. Am nächsten Tag war kein weiteres Luftleck nachweisbar. Sie erhielt Kortikosteroide, Antibiotika, Rekonvaleszenten-Plasma und Tocilizumab als Off-Label-Anwendung. Nach Besserung des Zustands und Extubation wurde die Thoraxdrainage an Tag 14 entfernt. Schließlich wurde die Patientin von der Sauerstofftherapie entwöhnt. Sie befindet sich derzeit in einer stationären Rehabilitationsbehandlung.

\section{Fall 5}

Eine 47-jährige Frau mit einem Meningeom, Diabetes, Hypothyreose und Adipositas (BMI: 33,6) in der Vorgeschichte stellte sich mit seit zwei Wochen bestehendem trockenen Husten, Fieber und Erschöpfung vor. Bei der Vorstellung hatte sie Fieber, Tachypnoe sowie eine Hypoxie, und sie benötigte Sauerstoff über eine HFNC
(SF-Quotient: 92). Bei den Laborbefunden waren eine Lymphopenie und erhöhte Entzündungsmarker auffällig. Die CT-Angiographie des Thorax zeigte eine Sattelembolie der Lunge, bilaterale Konsolidierungen und Milchglastrübungen. Sie erhielt Breitspektrum-Antibiotika, Kortikosteroide, eine kontinuierliche intravenöse Antikoagulation mit Heparin und im weiteren Verlauf Tocilizumab als Off-Label-Anwendung. Drei Tage später entwickelte die Patientin eine zunehmende Hypoxie mit erhöhter Atemarbeit, so dass eine mechanische Beatmung erforderlich war. Die Beatmungseinstellungen waren wie folgt: AF 18/min, TV: 400 $\mathrm{ml}, \mathrm{FiO}_{2}: 60 \%$ und PEEP $10 \mathrm{~cm} \mathrm{H}_{2} \mathrm{O}$. Der inspiratorische Spitzendruck betrug $27 \mathrm{~cm} \mathrm{H}_{2} \mathrm{O}$ und der Plateaudruck $23 \mathrm{~cm} \mathrm{H}_{2} \mathrm{O}$. An Tag 12 des Krankenhausaufenthalts wurde festgestellt, dass die Patientin zunehmend hypoxisch war und eine höhere inspiratorische Sauerstoffkonzentration bei der Beatmung benötigte. In der RT fand sich ein bilateraler Pneumothorax, der auf der rechten Seite größer war als auf der linken Seite. Daraufhin wurde bei der Patientin über einen offenen chirurgischen Zugang rechtsseitig eine Thoraxdrainage gelegt, woraufhin sich der Pneumothorax innerhalb von zwei Tagen zurückbildete. Der Krankheitsverlauf wurde durch eine disseminierte intravasale Gerinnung (disseminated intravascular coagulation, DIC), Nierenversagen und eine beatmungsassoziierte Pneumonie verkompliziert. Im weiteren Verlauf entwickelte sich ein Multiorganversagen. Die Patientin verstarb an einem Herzstillstand als Folge des zunehmenden akuten Atemnotsyndroms und eines septischen Schocks.

\section{Fall 6}

Bei der Patientin handelte es sich um eine 76-jährige Frau mit Lungensarkoidose, Adipositas (BMI: 43,15) und Bluthochdruck in der Vorgeschichte, die sich initial wegen einer progredienten Muskelschwäche vorstellte, die auf eine sensomotorische Polyneuropathie und Myopathie hindeutete. Wegen zunehmender Hyperkapnie und Somnolenz sowie der bestehenden Muskelschwäche war eine mechanische Beatmung mit anschließender Anlage einer Tracheostomie erforderlich. Während des Krankenhausaufenthalts entwickelte die Patientin anhaltendes Fieber sowie eine Hypoxie (SF-Quotient: 155 und PF-Quotient: 143). In den bildgebenden Untersuchungen des Thorax fanden sich bilaterale Konsolidierungen, die an der Lungenbasis besonders ausgeprägt waren, sowie atelektatische Veränderungen auf der linken Seite. Der Test aufCOVID-19 fiel positiv aus. Der Krankheitsverlauf wurde durch eine bilaterale tiefe Venenthrombose der unteren Extremität, eine beatmungsassoziierte Pneumonie durch Pseudomonas, ein Bakteriämie, Fungämie und einen septischen Schock verkompliziert. Nach einem längeren Krankheitsverlauf und trotz gleichbleibender Beatmungseinstellungen (AF: 14/min, TV: $450 \mathrm{ml}, \mathrm{FiO}_{2}: 100 \%$ und PEEP: $12,5 \mathrm{~cm} \mathrm{H}_{2} \mathrm{O}$ mit einem beobachteten PIP von $20 \mathrm{~cm} \mathrm{H}_{2} \mathrm{O}$ und einem Plateaudruck von $16 \mathrm{~cm}$ $\mathrm{H}_{2} \mathrm{O}$ ) entwickelte die Patientin einen rechtsseitigen Pneumothorax. Daraufhin wurde über einen offenen chirurgischen Zugang eine Thoraxdrainage gelegt, was zu einer Expansion der Lunge führte. Leider kam es am folgenden Tag zu einem Herzstillstand. Trotz Wiederbelebungsmaßnahmen verstarb die Patientin.
42

Kompass Pneumol 2021;9:39-46 DOI: $10.1159 / 000513501$ 
Tab. 3. Zusammenfassung der Literaturberichte über Pneumothorax bei COVID-19-Patienten; Daten zum Vergleich der klinischen/radiologischen Merkmale und der klinischen Outcomes

\begin{tabular}{|c|c|c|c|c|c|c|c|c|}
\hline & $\begin{array}{l}\text { Alter in J./ } \\
\text { Geschlecht }\end{array}$ & CT-Charakteristika & Komplikation & Risikofaktoren & $\begin{array}{l}\text { Zeit bis zum Auftreten, } \\
\text { Tage }\end{array}$ & $\begin{array}{l}\text { Thorax- } \\
\text { drainage }\end{array}$ & $\begin{array}{l}\text { Zeit bis zur } \\
\text { vollständigen } \\
\text { Rückbildung } \\
\text { (Tage) }\end{array}$ & Outcomes \\
\hline Zhou et al. [3] & $38 / m$ & $\begin{array}{l}\text { Bilaterale GGOs und } \\
\text { Konsolidierungen in den } \\
\text { Unterlappen }\end{array}$ & Pneumomediastinum & Keine & 11 & & 14 & Überlebt \\
\hline Wang et al. [5] & $36 / w$ & $\begin{array}{l}\text { Bilaterale fleckförmige GGOs und } \\
\text { Konsolidierungen }\end{array}$ & Pneumomediastinum & NIV & $\begin{array}{l}\text { Tag } 0 \text { bis } 12 \text { Tage nach } \\
\text { Beginn der Symptome }\end{array}$ & & & $\begin{array}{l}\text { Verstorben infolge } \\
\text { ARDS }\end{array}$ \\
\hline Sun et al. [6] & $38 / \mathrm{m}$ & $\begin{array}{l}\text { Fleckförmige periphere GGOs } \\
\text { Progression zu Konsolidierungen } \\
\text { und Bullae }\end{array}$ & $\begin{array}{l}\text { Mediastinales } \\
\text { Emphysem, Riesenbulla, } \\
\text { Pneumothorax }\end{array}$ & NIV & $\begin{array}{l}\text { Pneumomediastinum: } \\
7 \text { Tage } \\
\text { Bullae: } 21 \text { Tage } \\
\text { PNX: } 30 \text { Tage }\end{array}$ & Keine & & \\
\hline Aiolfi et al. [4] & $\begin{array}{l}56 / \mathrm{m} \\
70 / \mathrm{m}\end{array}$ & Bilaterale periphere GGOs & Pneumothorax & $\begin{array}{l}\text { Invasive } \\
\text { mechanische } \\
\text { Ventilation } \\
\text { Präexistentes } \\
\text { Emphysem }\end{array}$ & $\begin{array}{l}2 \text { und } 5 \text { Tage nach } \\
\text { Intubation }\end{array}$ & $\mathrm{Ja}$ & & $\begin{array}{l}\text { Wegen eines } \\
\text { persistierenden } \\
\text { Pneumothorax } \\
\text { wurden eine } \\
\text { Thorakotomie und } \\
\text { Bleb-Resektion } \\
\text { durchgeführt }\end{array}$ \\
\hline Liu et al. [11] & $38 / \mathrm{m}$ & $\begin{array}{l}\text { Bilaterale fleckförmige GGOs und } \\
\text { Konsolidierungen, Progression zu } \\
\text { zystischen Veränderungen }\end{array}$ & Pneumothorax & Keine & 26 & Keine & 5 & Überlebt \\
\hline $\begin{array}{l}\text { Wang et al. } \\
\text { Wang et al. [10] }\end{array}$ & $62 / m$ & $\begin{array}{l}\text { Bilateral Areale mit GGOs in der } \\
\text { Peripherie }\end{array}$ & $\begin{array}{l}\text { Pneumomediastinum } \\
\text { Pneumothorax } \\
\text { Subkutanes Emphysem }\end{array}$ & Keine & 20 & Keine & 16 & Überlebt \\
\hline
\end{tabular}

\section{Diskussion}

Eine pulmonale Grunderkrankung ist der primäre Risikofaktor für das Auftreten eines sekundären Spontanpneumothorax. Dazu gehören die chronisch obstruktive Lungenerkrankung (chronic obstructive pulmonary disease, COPD) mit Emphysem, zystische Fibrose, Tuberkulose, Lungenkrebs, HIV-assoziierte Pneumocystis-jiroveci-Pneumonie (PJP) und andere zystische Lungenerkrankungen $[1,2,12]$.

Bei Patienten mit einer COVID-19-Infektion kann es zu einer schweren Pneumonie kommen, die zu einem akuten Atemnotsyndrom (acute respiratory distress syndrome, ARDS) führt. Röntgenologisch ist die Erkrankung durch Milchglastrübungen gekennzeichnet, die sich im weiteren Verlauf zu konsolidierenden Veränderungen und in späteren Erkrankungsstadien zu fibrotischen Veränderungen entwickeln $[8,13]$. Man nimmt an, dass beim Spontanpneumothorax ähnliche Veränderungen, darunter schwere Lungenschädigungen und diffuse Alveolarschäden, beteiligt sind, die ein schweres akutes respiratorisches Syndrom (severe acute respiratory syndrome, SARS) verkomplizieren [14]. Neben einer möglichen Überdehnung der Alveolen durch die mechanische Beatmung können diese Veränderungen das Risiko für die Patienten, einen Pneumothorax zu entwickeln, erhöhen. Tabelle 3 enthält eine Zusammenfassung der Literaturberichte, die über Pneumothorax bei COVID-19-Patienten veröffentlicht wurden.

In dieser Fallserie identifizierten wir sechs von 902 Patienten mit COVID-19-Pneumonie, die einen Spontanpneumothorax entwickelten. Damit liegt die Inzidenz $(0,66 \%)$ niedriger als in den kürzlich veröffentlichten Berichten $[7,8]$.
Die mechanische Beatmung scheint ein Hauptrisikofaktor für die Entwicklung eines Pneumothorax bei COVID-19-Pneumonie zu sein. Aiodfi und Kollegen berichteten über zwei Fälle von Patienten mit COVID-19-Pneumonie, die unter der mechanischen Beatmung einen persistierenden Pneumothorax entwickelten [4]. Dagegen berichteten Wang et al. über einen Patienten, der ohne mechanische Beatmung einen Spontanpneumothorax, ein Pneumomediastinum und ein subkutanes Emphysem entwickelte [10]. Ebenso berichteten Zhou et al. über einen Fall von Spontanpneumomediastinum bei einem spontanatmenden COVID-19-Patienten [3]. Man nimmt an, dass die hohe Inzidenz von Pneumothorax bei mechanisch beatmeten Patienten bei Patienten mit ARDS sogar noch höher ist und zwischen $14 \%$ und $87 \%$ beträgt. Es besteht eine direkte Korrelation mit der Schwere und Dauer des ARDS sowie dem Baro- und Volumentrauma, die Folge der mechanischen Beatmung sind. Diese treten insbesondere in Fällen auf, in denen hohe inspiratorische Spitzendrücke (PIP) (>40$50 \mathrm{~cm} \mathrm{H} \mathrm{H}_{2} \mathrm{O}$ ), ein hoher positiver endexspiratorischer Druck (PEEP) sowie hohe Tidal- und Atemminutenvolumina vorliegen $[15,16,17]$. Darüber hinaus spiegelt das ARDS eine heterogene Gruppe von Erkrankungen wider, bei denen ein Nebeneinander von relativ gesunden Alveolen gemischt mit erkrankten Alveolen besteht. Häufig neigen die abhängigen Lungenabschnitte zur Konsolidierung infolge eines interstitiellen Ödems und es handelt sich um Areale mit verminderter Compliance. Bei Rekrutierungsmanövern im Rahmen des ARDS-Managements kommt es zu einer Überdehnung von «normalen» nicht abhängigen Lungenabschnitten mit vergleichsweise höherer Compliance und geringerem Atemwegswiderstand. Aufgrund der ungleichmäßigen Verteilung von Volumen und Druck durch das Beatmungsgerät 

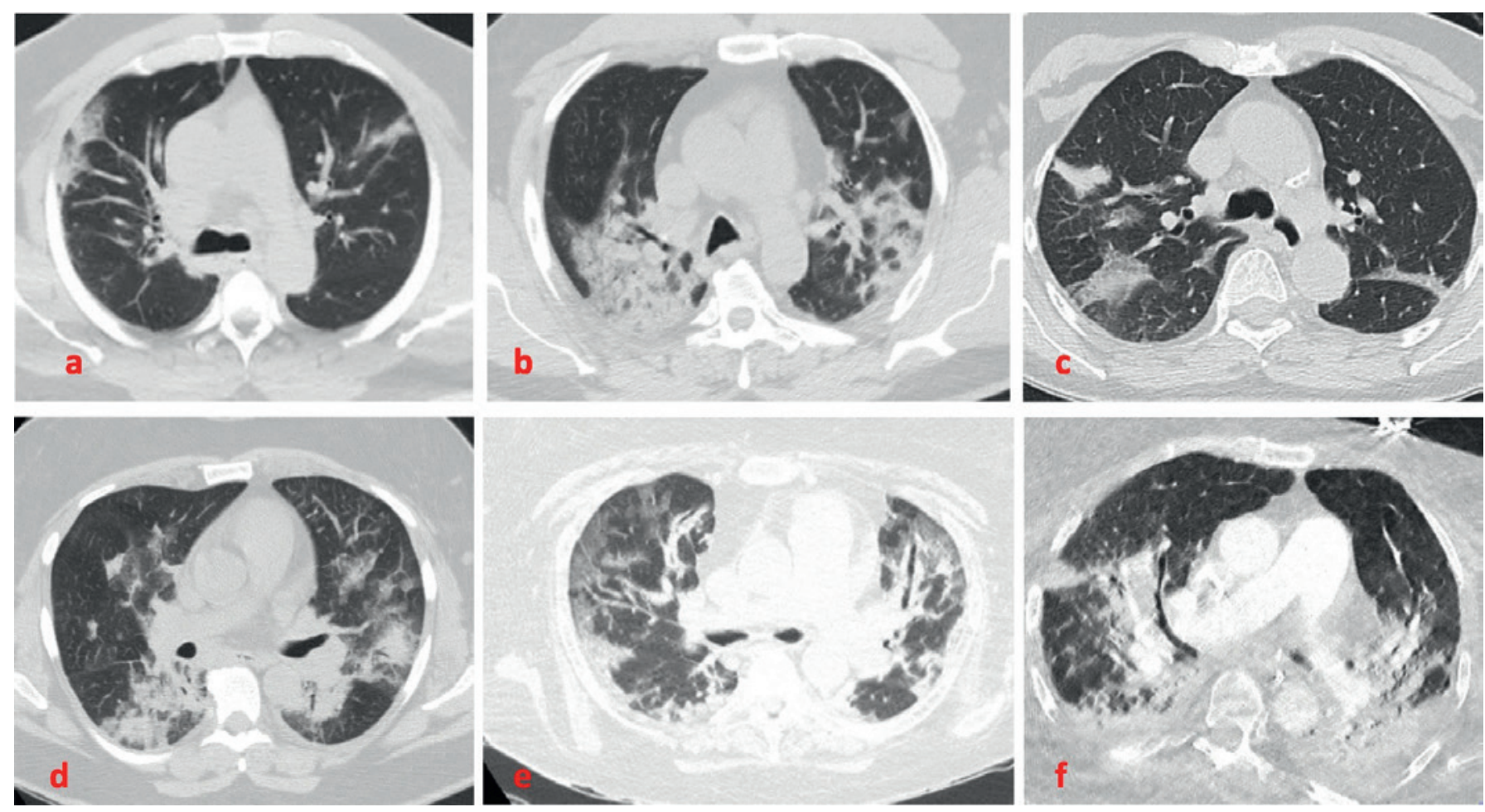

Abb. 1. Baseline-CT-Aufnahmen des Thorax. Diese zeigen Milchglastrübungen, Konsolidierungen und Crazy-Paving-Muster, überwiegend bilateral und hauptsächlich in den posterioren und peripheren Lungenregionen. Von links oben: (a) Fall 1, (b)Fall 2, (c) Fall 3, (d) Fall 4, (e) Fall 5, (f) Fall 6.

und die dadurch bedingten erhöhten Scherkräfte können die Alveolen rupturieren. Vier unserer sechs Patienten (66\%) wurden mechanisch beatmet. Allerdings wiesen sie eine höhere Compliance der Lunge auf, was sich in den niedrigeren inspiratorischen Spitzendrücken und Plateaudrücken zeigte. In einem kürzlich erschienenen Bericht über Patienten mit COVID-19-Pneumonie wird die Hypothese aufgestellt, dass eine Untergruppe von Patienten eine nahezu normale Compliance hat [18].

Gattinoni et al. stellten fest, dass die Inzidenz des Pneumothorax bei ARDS-Patienten, die lange mechanisch beatmet werden, höher ist ( $87 \%$ vs. $30 \%$ bei Patienten mit $\geq 2$-wöchiger mechanischer Beatmung bzw. $<1$-wöchiger Beatmung). Daneben kam es auch bei Patienten mit Bullae und niedriger Compliance der Lunge häufiger zu einem Pneumothorax - ein Ergebnis, das auf unsere Patienten nicht zutrifft [18]. Unabhängig davon ist es entscheidend, dass lungenprotektive Beatmungsstrategien mit geringen Volumina und Druckbegrenzung zum Einsatz kommen, um Baro- und Volumentraumata zu minimieren. Unserer Ansicht nach können diese Maßnahmen auch bei Patienten mit hoher Compliance und schwerer Hypoxämie von Nutzen sein. Zur Bestätigung dieser Hypothese sind weitere Studien erforderlich, in denen diese spezielle Patientenpopulation und die Effekte protektiver mechanischer Beatmungsstrategien untersucht werden. Außerdem ist zu berücksichtigen, dass bis jetzt keine eindeutigen Leitlinien über den Zeitpunkt und die Einstellungen bei der mechanischen Beatmung von Patienten mit COVID-19-Pneumonie existieren. Eine sicherere Alternative zur Vermeidung potenziel- ler Komplikationen der mechanischen Beatmung bei diesen Patienten könnte die High-Flow-Sauerstofftherapie darstellen.

Ein weiterer möglicher auslösender Faktor ist anhaltender Husten, der ein häufiges Symptom der COVID-19-Krankheit ist [7, 19, 20]. Vier von sechs Patienten gaben bei der Erstvorstellung Husten als Hauptsymptom an. Da Husten eine plötzliche Verlängerung und Verkürzung der Lungengefäße und der zugehörigen Bronchien während der Respiration verursacht, kann er den Luftaustritt aus den Alveolen verstärken und den «Train of Bubbles» entlang der Gefäßscheiden weiterbewegen [21].

Bildgebende Untersuchungen bei Baseline können ein Prädiktor für das Auftreten eines Pneumothorax sein. Ein kürzlich veröffentlichter Artikel berichtete über zwei Patienten mit zystischen Lungenläsionen im Zusammenhang mit COVID-19-Erkrankung, von denen einer einen Pneumothorax entwickelte [11]. Dieser Fall ähnelt Fällen von Spontanpneumothorax bei HIV-Patienten und PJP-Pneumonie [22]. Ein anderer Fallbericht beschrieb einen Patienten ohne pulmonale Grunderkrankung bei Baseline, der als Folge einer COVID-19-Infektion eine Riesenbulla entwickelte, die schließlich rupturierte und zu einem Pneumothorax führte [6]. Bei unseren Patienten fanden sich unterschiedlich stark ausgeprägte Milchglastrübungen, Konsolidierungsareale, Crazy-Paving-Muster und Atelektasen, was den Angaben in der Literatur entspricht $[13,23]$. In unserer Klinik erfolgt bei jedem Patienten mit COVID-19-Krankheit eine CT-Untersuchung des Thorax bei der Aufnahme (Abb. 1) und täglich wird eine RoutineRöntgenaufnahme des Thorax erstellt; diese dienen als Hilfe für
44

Kompass Pneumol 2021;9:39-46

DOI: 10.1159/000513501 

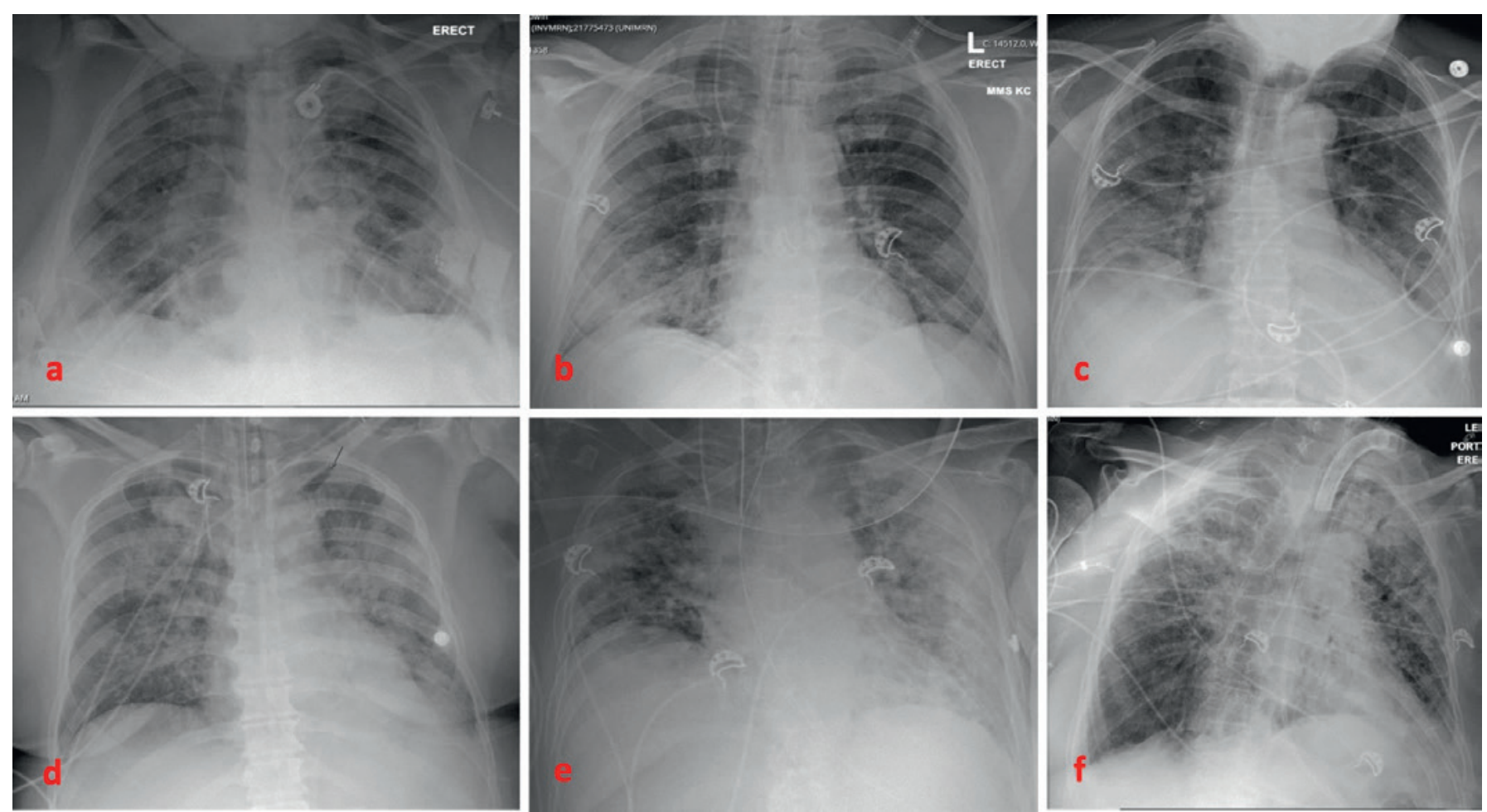

Abb. 2. Interims-Röntgenaufnahmen des Thorax der einzelnen Patienten vor Auftreten des Pneumothorax. Von links oben: (a) Fall 1 (b) Fall 2, (c) Fall 3, (d) Fall 4, (e) Fall 5, (f) Fall 6.
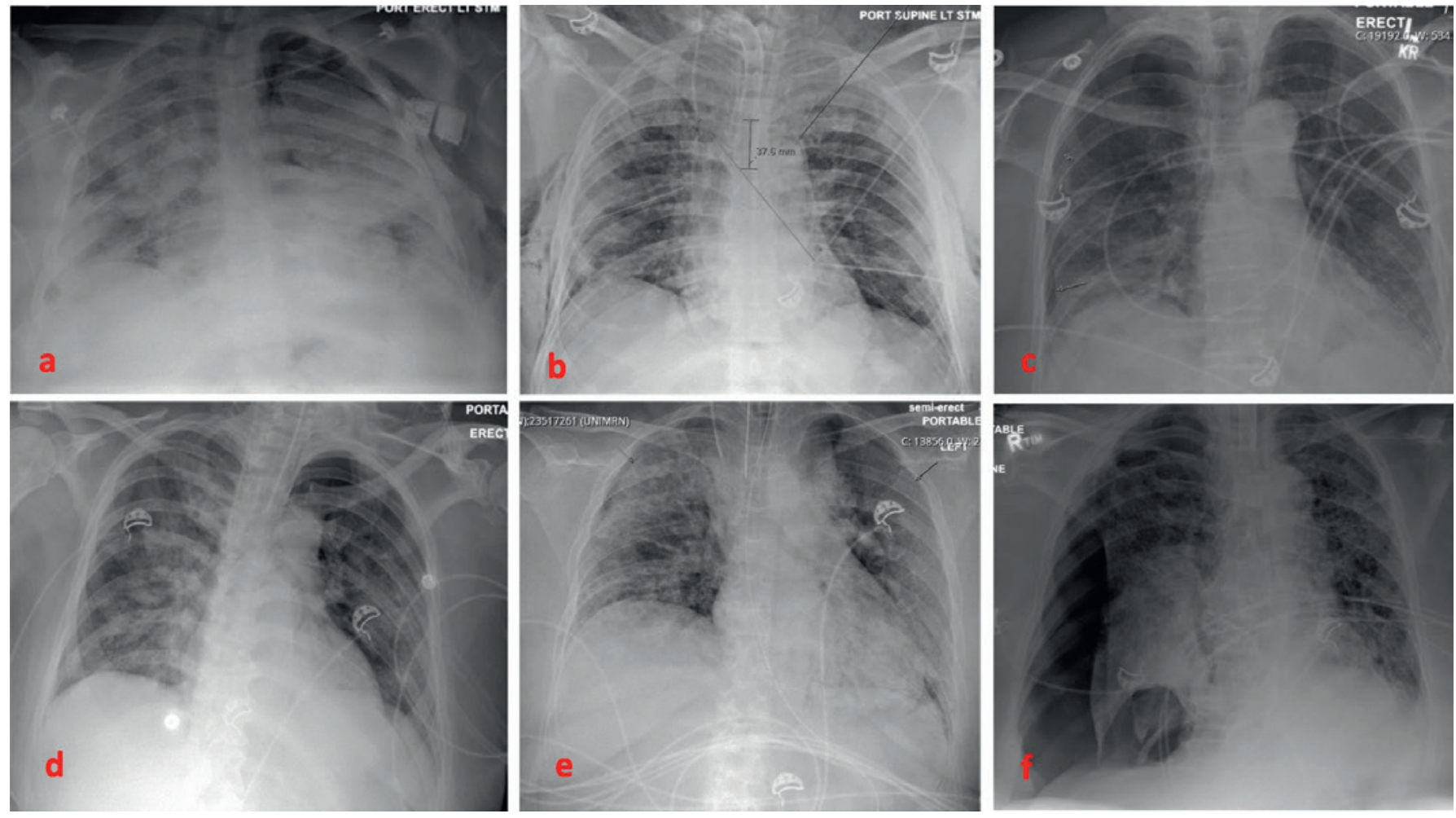

Abb. 3. Thorax-Röntgenaufnahmen der einzelnen Patienten, die den Pneumothorax zeigen. Von links oben: (a) Fall 1 Groß, links. (b) Fall 2 nach Pneumothorax-Entwicklung und Anlage von Drainagen. Restbefund eines rechtsseitigen subkutanen Emphysems. (c) Fall 3 Mäßig, rechts (d) Fall 4. Klein, apikal links (e) Fall 5 Klein, bilateral (f) Fall 6 Groß, rechts. 
die klinischen Entscheidungen und das medizinische Management. Bei den meisten Patienten gelang es, die radiologische Progression der Parenchymbefunde vor der Entwicklung eines Pneumothorax zu erfassen (Abb. 2 und 3).

Ein wichtiges Ergebnis unserer Studie war, dass nahezu alle Patienten, die einen Spontanpneumothorax entwickelten, eine Lymphopenie und erhöhte Werte für die Entzündungsmarker wie CRP, LDH, Ferritin, D-Dimere und IL-6 aufwiesen (Tab. 2). Dies entspricht den Ergebnissen von kürzlich veröffentlichten Studien, in denen die möglichen Mechanismen einer COVID-19-induzierten Lungenschädigung untersucht wurden. Es wird vermutet, dass ein Zytokinsturm an der Pathophysiologie der Erkrankung beteiligt ist. Diese Form der hyperaktiven und fehlgesteuerten Immunreaktion kann zu einem hyperinflammatorischen ARDS führen und ist mit einer kritischen Erkrankung und erhöhter Mortalität verbunden [24, 25, 26]. Darüber hinaus fanden sich Thrombosen und Mikroangiopathien im Lungengewebe von $\mathrm{Pa}$ tienten mit ARDS, die an einer COVID-19-Infektion verstorben waren. Man nimmt an, dass dies bei der Lungenschädigung eine Rolle spielt; allerdings sind weitere Studien erforderlich, in denen die Auswirkungen dieser Ergebnisse untersucht werden [27]. Sofern keine Kontraindikationen bestanden, erhielten alle Patienten mit einer COVID-19-Infektion in unserer Klinik eine prophylaktische oder therapeutische Antikoagulation. Die Auswahl der Art und Dosis der Antikoagulation erfolgte im Rahmen einer interdisziplinären Beratung, an der auch Hämatologen teilnahmen. Bei der Entscheidung wurden die Entzündungsmarkerwerte, insbesondere die D-Dimer-Konzentration, einbezogen.

Und schließlich schlossen wir den Fall eines Patienten ein, bei dem es während der Einbringung einer perkutanen Tracheostomiekanüle zu einem scheinbar spontanen bilateralen Pneumothorax gekommen war. Man nimmt an, dass diese Komplikation durch eine Verletzung der anterioren oder posterioren Trachealwand bedingt ist, die zu einer Schädigung der Pleurahöhle führt $[28,29]$. Unser Patient wies keine erkennbaren Trachealrupturen auf. Unserer Ansicht nach handelte es sich bei dem zugrunde liegenden Mechanismus für den bilateralen Pneumothorax höchstwahrscheinlich um eine Kombination aus Überdruckbeatmung, Schleimretention und entzündetem Lungenparenchym infolge der COVID-19-Erkrankung. Eine heterogene Überdehnung der Alveolen durch Schleimretention und/oder die Konsolidierungs- phase einer viralen Pneumonie bei COVID-19 können das Risiko für einen Spontanpneumothorax erhöhen. Eine Überdehnung kann zudem in entzündeten Alveolen (Areale mit Milchglastrübungen) auftreten. Zwar handelt es sich um eine seltene Komplikation bei der Anlage einer Tracheostomie, doch sollte sich der Arzt, der den Eingriff durchführt, darüber im Klaren sein, dass das Risiko für einen Pneumothorax bei COVID-19-Patienten mit diffuser Lungenparenchymerkrankung potenziell erhöht ist, und beim Umgang mit den Atemwegen dieser Patienten ist besondere Vorsicht geboten.

\section{Schlussfolgerung}

Der Spontanpneumothorax ist eine seltene Komplikation der COVID-19-Viruspneumonie, die jederzeit während des Krankheitsverlaufs auftreten kann. Bei Patienten mit Milchglastrübungen und Konsolidierungen bei Baseline sowie Patienten, die mechanisch beatmet werden, scheint ein erhöhtes Risiko zu bestehen. Kliniker sollten im Hinblick auf die Diagnose und Behandlung dieser Komplikation wachsam sein.

\section{Zustimmung zur Veröffentlichung}

Eine Einverständniserklärung der Patienten oder der gesetzlichen Vertreter für die Veröffentlichung der jeweiligen Fallberichte und der dazugehörigen Bilder wurde eingeholt.

\section{Disclosure Statement}

Die Autoren erklären, dass keine Interessenskonflikte bestehen.

\section{Lizenzangabe}

Massa Zantah, Eduardo Dominguez Castillo, Ryan Townsend, Fusun Dikengil, Gerard J. Criner: Pneumothorax in COVID-19 disease - incidence and clinical characteristics. Respir Res. 2020;21(1):236. (DOI: DOI 10.1186/ s12931-020-01504-y). ${ }^{\circledR} 2020$ The Authors. (Übersetzung; Abkürzungen, Danksagung, Autorenbeiträge, Finanzielle Unterstützung, Verfügbarkeit von Daten und Materialien, Genehmigung durch die Ethikkommission und Einwilligung in die Teilnahme und Zustimmung zur Veröffentlichung gekürzt), lizensiert unter CC BY 4.0 (https://creativecommons.org/licenses/by/4.0/deed.de).

\section{Literatur}

Die Literatur ist unter www.karger.com/Article/Fulltext/513501 abrufbar. 